Supporting Information for:

\title{
Moisture-Driven Formation and Growth of Quasi-2D Organolead Halide Perovskite Crystallites
}

\author{
Bryan R. Wygant, ${ }^{a}$ Geoff T. Geberth, ${ }^{a}$ Alexandre Z. Ye, ${ }^{b}$ Andrei Dolocan, ${ }^{c}$ Daniel E. Cotton, ${ }^{a}$ Sean T. Roberts, ${ }^{a}$ \\ David A. Vanden Bout, ${ }^{a}$ C. Buddie Mullins ${ }^{a, b, c, *}$ \\ ${ }^{a}$ Department of Chemistry, ${ }^{b}$ McKetta Department of Chemical Engineering, ${ }^{c}$ Texas Material Institute; University of \\ Texas at Austin, Austin, TX 78712 \\ *corresponding author: mullins@che.utexas.edu
}




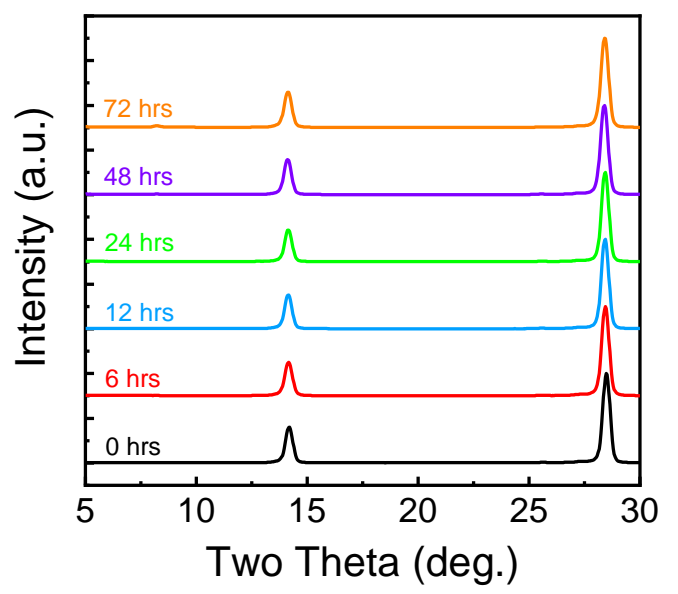

Figure S1 XRD spectra of an nBA-MAPI film over 72 hours of moisture exposure at $78 \% \mathrm{RH}$. 

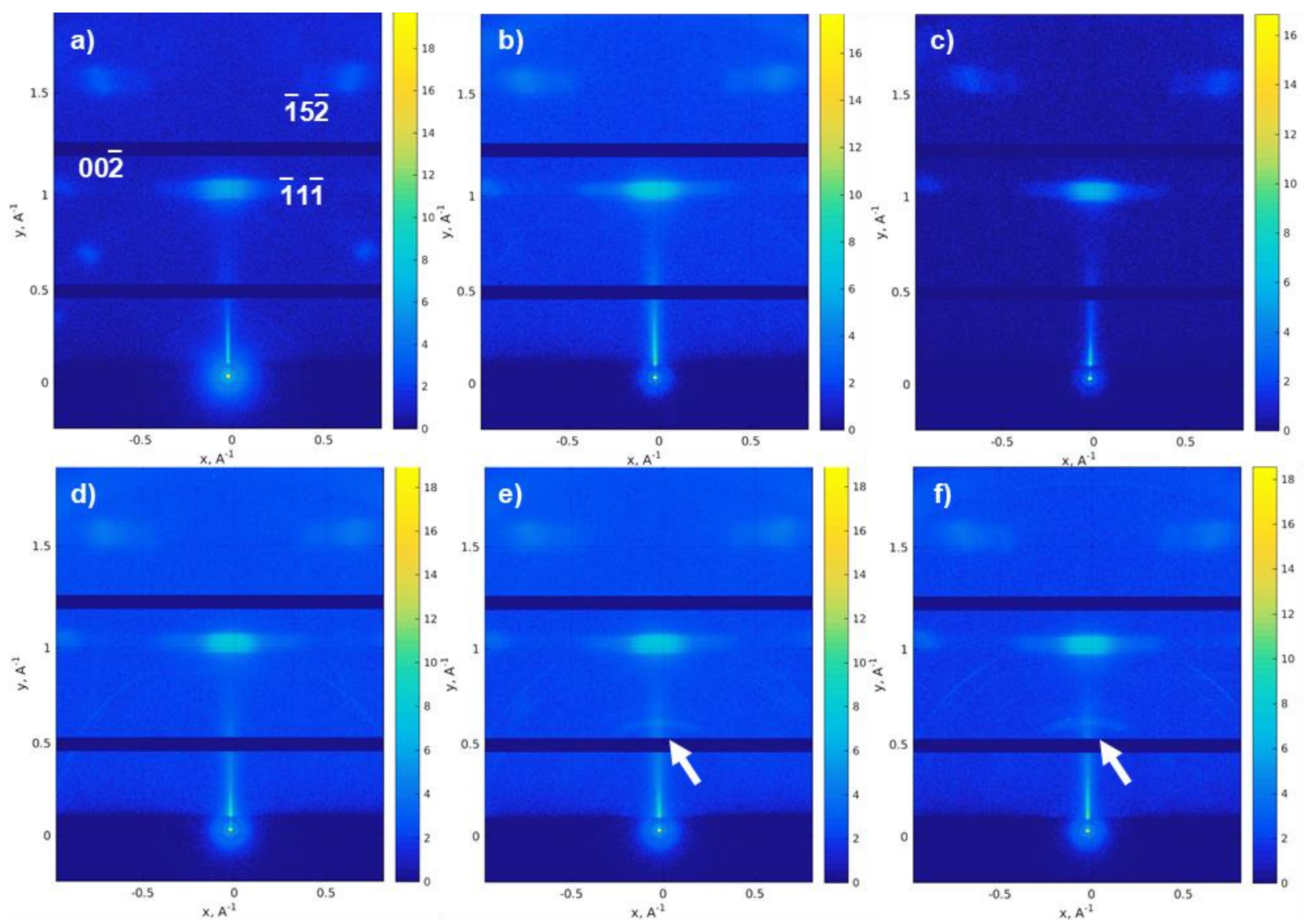

Figure S2 WAXS spectra of nBA-MAPI films at a) 0 hours, b) 6 hours, c) 12 hours, d) 24 hours, e) 48 hours, and f) 72 hours of exposure to $78 \% \mathrm{RH}$. All samples show well-oriented crystal structures, as indicated by the clear spots

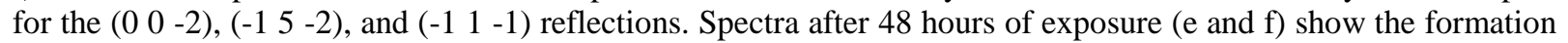
of rings at low- $q$ (indicated by white arrows), indicating the formation of un-oriented low- $n$ perovskite phases after sufficient exposure. 


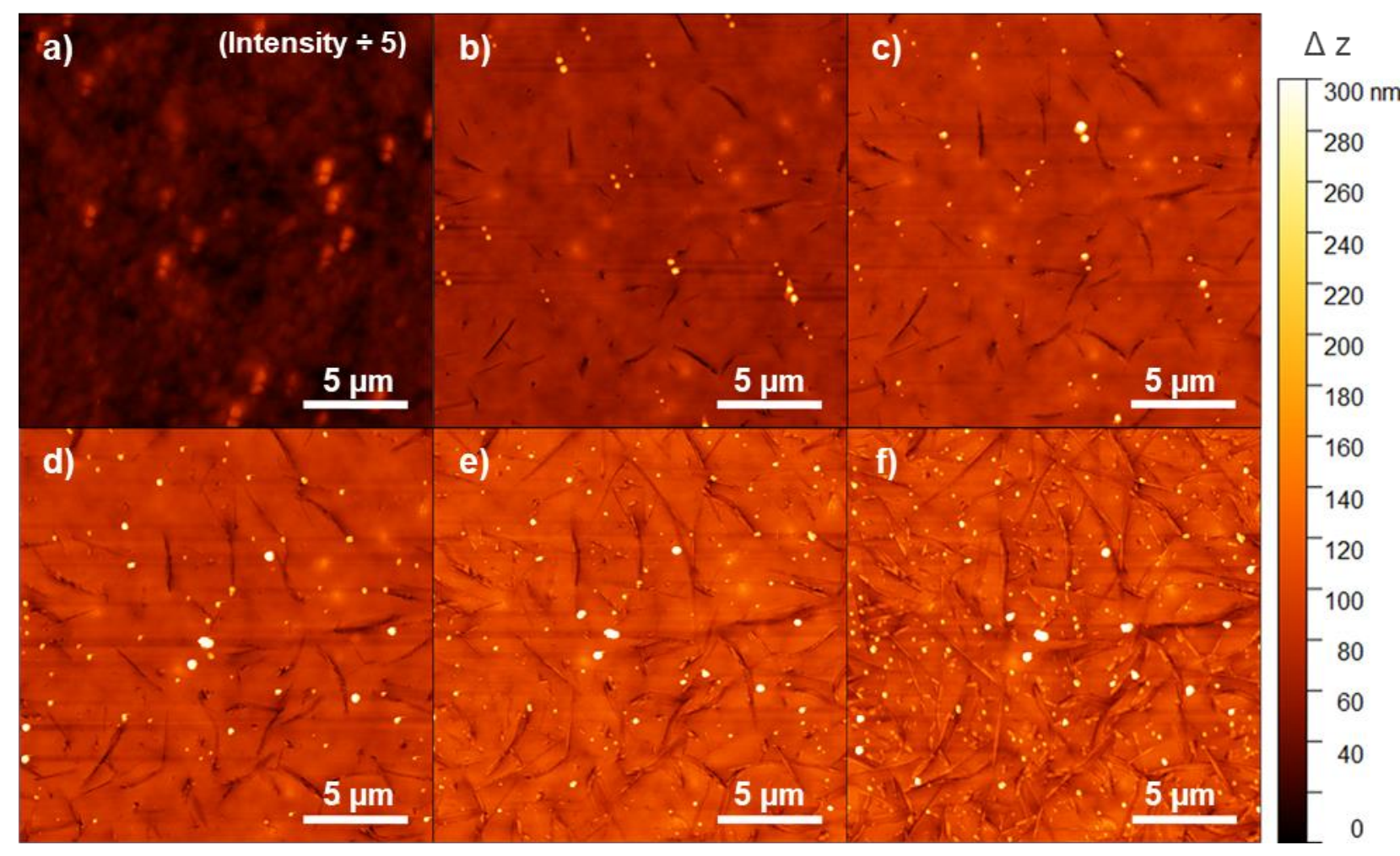

Figure S3 AFM images of a single area of an nBA-MAPI film acquired in situ over a) 0 hours, b) 6 hours, c) 12 hours, d) 24 hours, e) 48 hours, and f) 72 hours of exposure to $78 \% \mathrm{RH}$. Cracks initially form in the surface of the film after just 6 hours of exposure (b), and grow over time. Crystalline structures also appear between b) and c), and likewise continue to grow, covering the surface after 72 hours (f). 


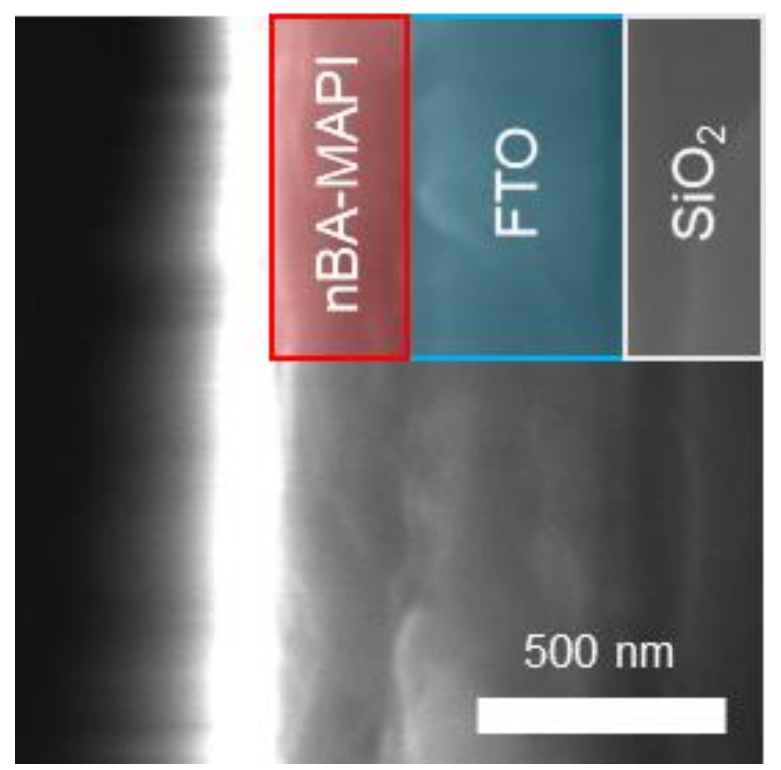

Figure S4 Cross-sectional SEM image of a typical nBAMAPI film showing an average thickness of roughly 350 nm. 

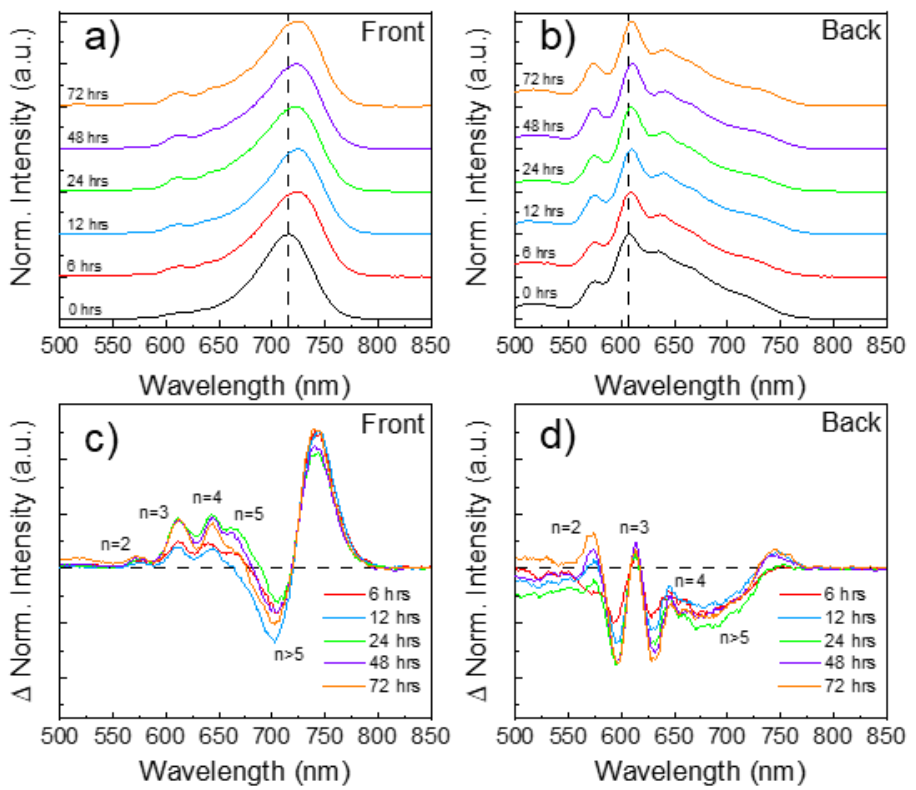

Figure S5 PL spectra taken from the front (a) and back (b) of an nBAMAPI film over the course of 72 hours of exposure to $78 \% \mathrm{RH}$. New peaks in the 575 to $650 \mathrm{~nm}$ range, corresponding to $n=2-5$ phases, appear on the front side (a) after exposure, and the primary peak at $715 \mathrm{~nm}$ shifts to $725 \mathrm{~nm}$. Similar, but smaller spectral changes are observed in b). Normalized PL difference plots from the front (c) and back (d) of the same film, showing the formation of low- $n$ species and a 3D-like species concurrent with the loss of the $n>5$ species with exposure. 


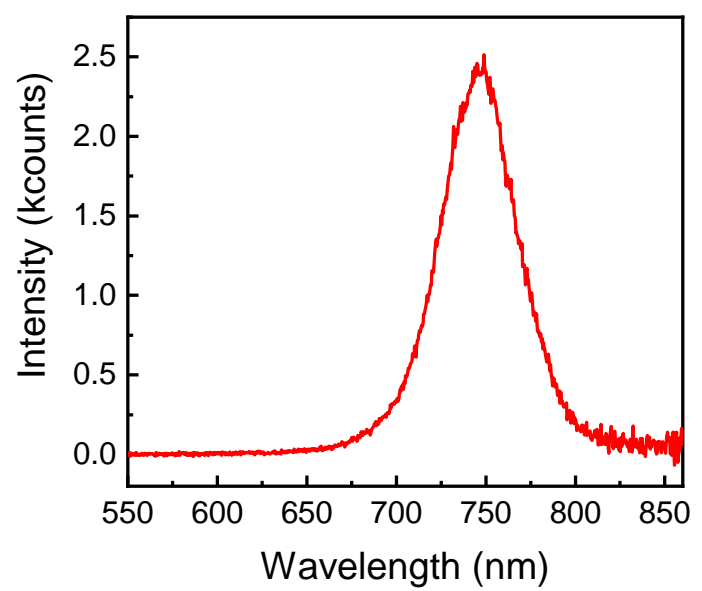

Figure S6 Point fluorescence spectrum from an nBAMAPI film showing a strong peak near $750 \mathrm{~nm}$. 


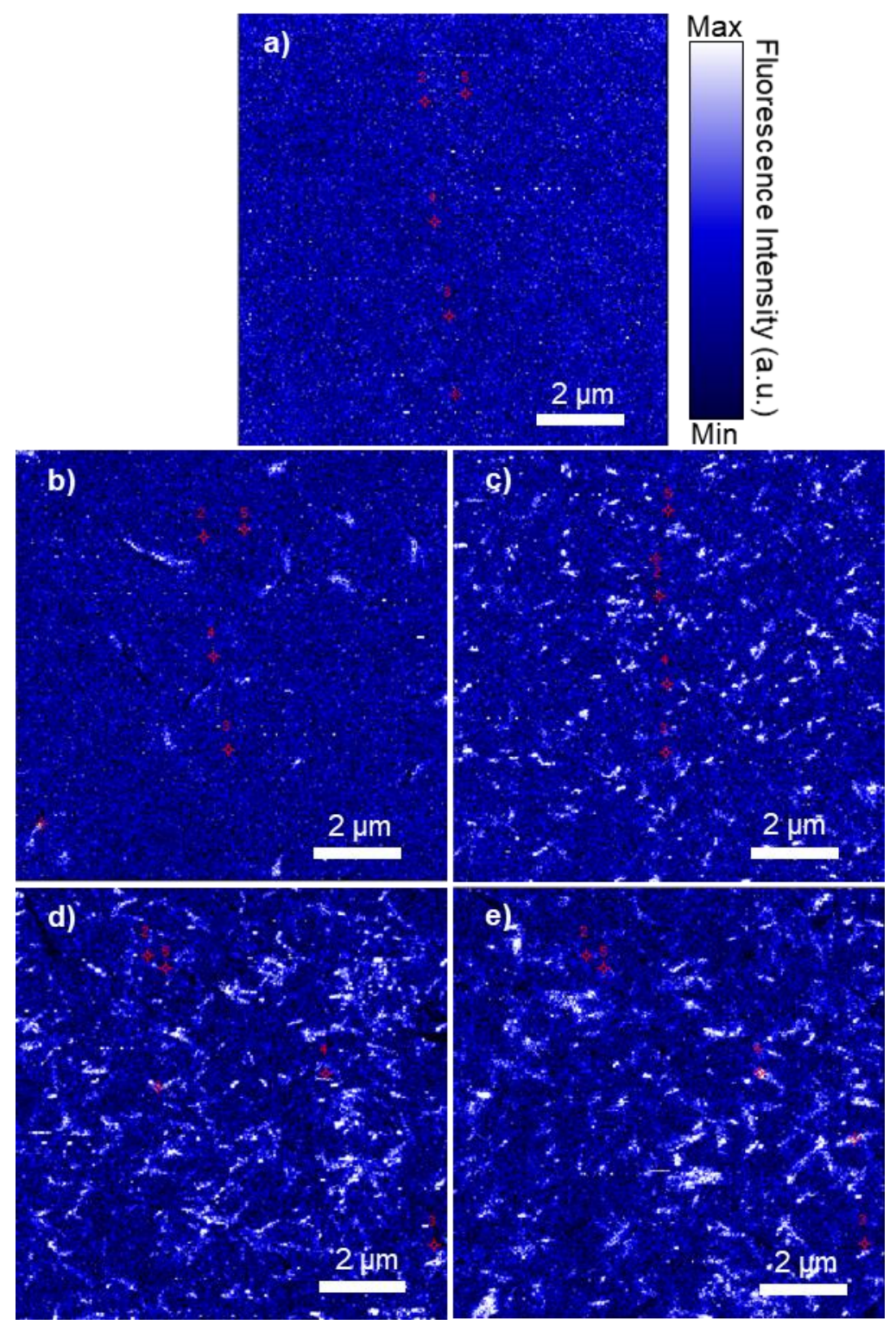

Figure S7 CFM images of a nBA-MAPI films at a) 0 hours, b) 12 hours, c) 24 hours, d) 48 hours, and e) 72 hours of exposure to $78 \% \mathrm{RH}$ with a $740 \mathrm{~nm}$ short-pass filter. After 12 hours (b) fluorescence from elongated structures on the film is observed, and the size and surface density of these structures increases with exposure. The red crosshairs and numbers are artifacts from the imaging software. 


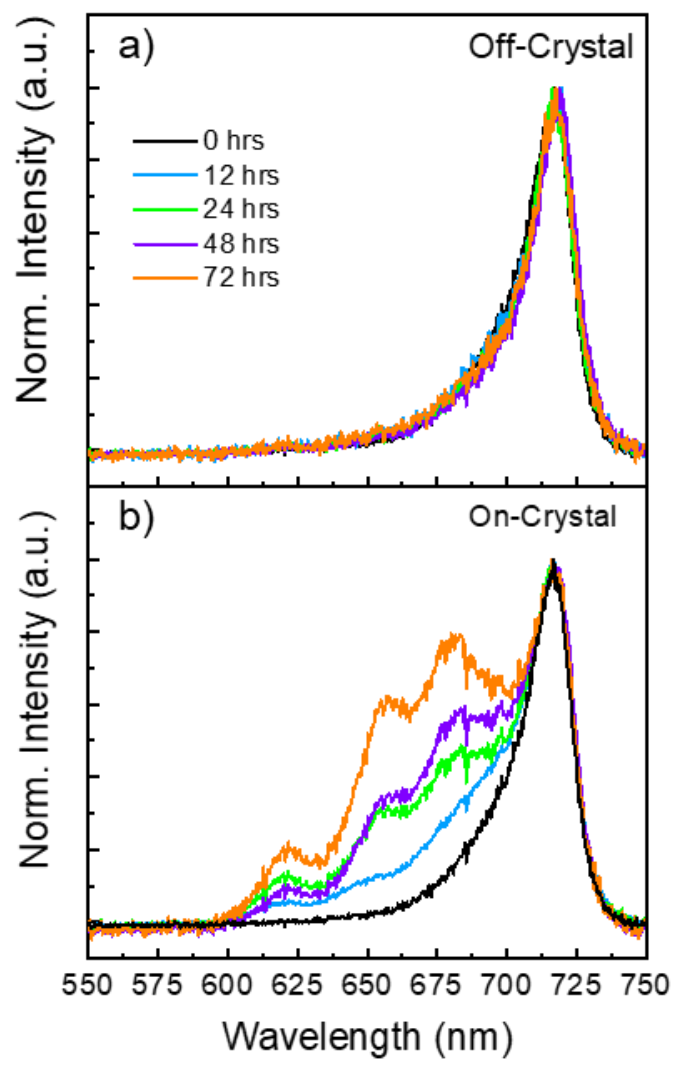

Figure S8 Average normalized point fluorescence spectra from CFM of areas off- (a) and on-crystal (b) taken from nBA-MAPI films exposed to $78 \% \mathrm{RH}$ over 72 hours. In a) only the shoulder of the large peak at 750 $\mathrm{nm}$ can be observed for all samples. In b), there is a clear increase in peaks at 620,660 , and $680 \mathrm{~nm}$ with exposure time, corresponding to formation of $n=3,4$, and 5 peaks, respectively. 


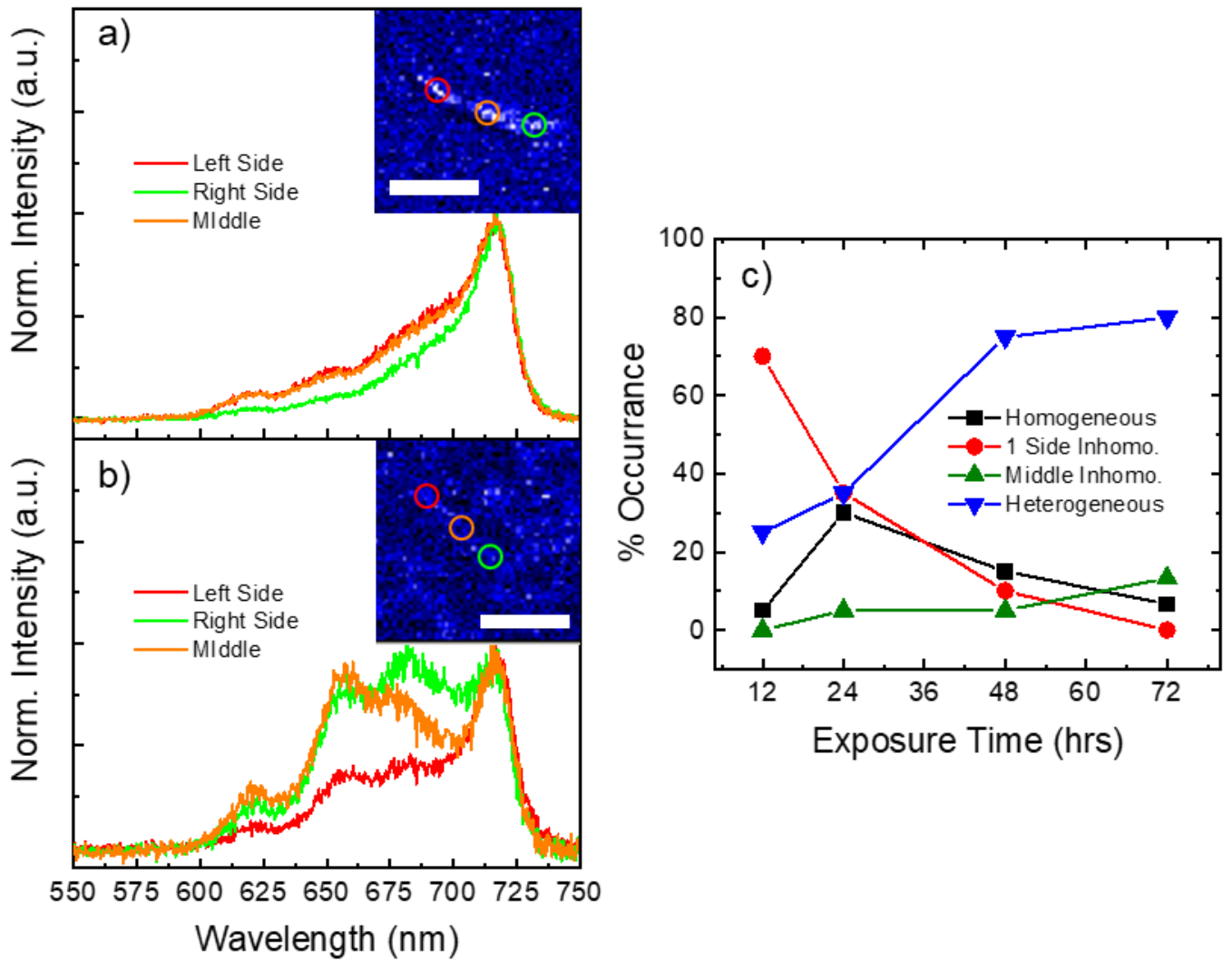

Figure S9 Point fluorescence spectra taken from three different locations of the same nBA-MAPI crystal using CFM at a) 12 hours and b) 72 hours of exposure to $78 \% \mathrm{RH}$, demonstrating differences in crystal homogeneity. The inset image in each provides an example of the locations sampled for each spectrum. Scale bar equal to $1 \mu \mathrm{m}$. c) Plot showing the prevalence of different levels of crystal homogeneity in nBAMAPI crystals over 72 hours of exposure. Over time, the overall homogeneity of the crystals decreases as spectra from different locations of the crystals begin to show varying quantities of different $n$-phases. 


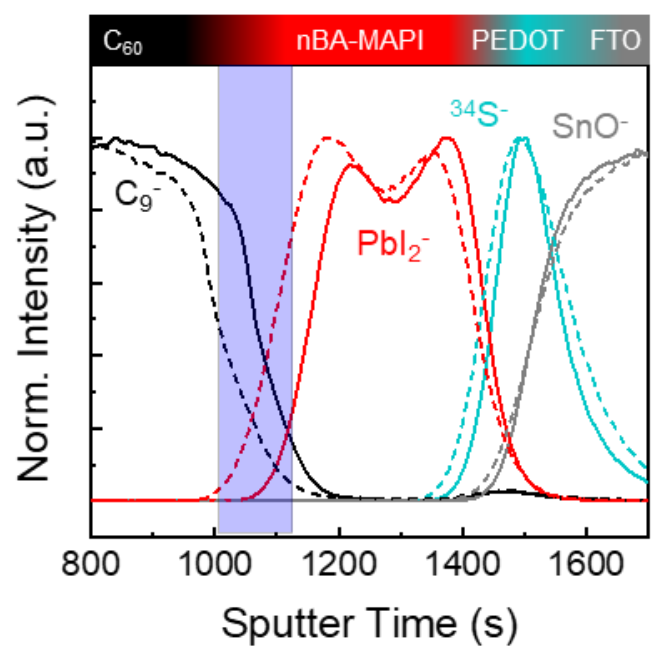

Figure S10 ToF-SIMS depth profile of various species from fresh (solid line) and exposed (dashed line) model PV devices. Exposed devices were left exposed to $78 \%$ RH for 24 hours prior to analysis. After exposure, the perovskite layer $\left(\mathrm{PbI}_{2}{ }^{-}\right)$shows an increase in width at the $\mathrm{C}_{60}$ interface, largely corresponding the hydration layer that has built up here (blue shaded region) and indicating moisture-related changes in the material. 

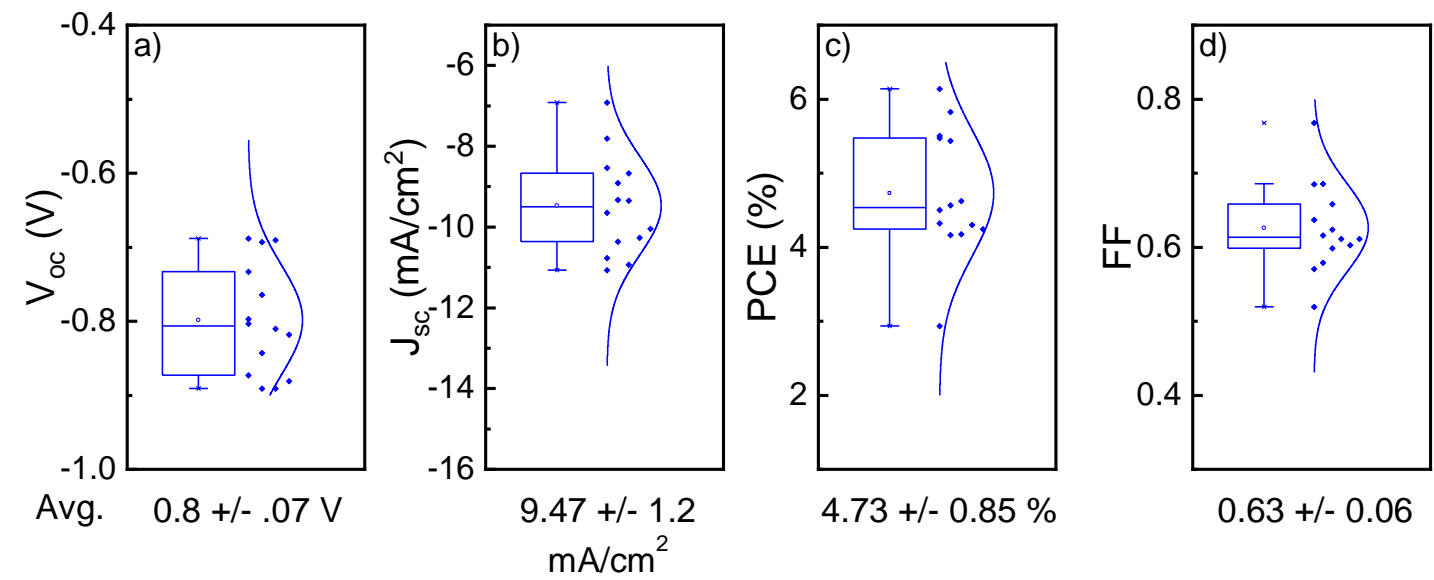

Figure S11 Initial device performance of a set of nBA-MAPI PV devices tested at $100 \mathrm{~mW} / \mathrm{cm}^{2}$ AM1.5G light, showing the (a) open-circuit voltage, (b) short-circuit current, (c) power conversion efficiency, and (d) fill factor. Results are adapted from ref. 1. 


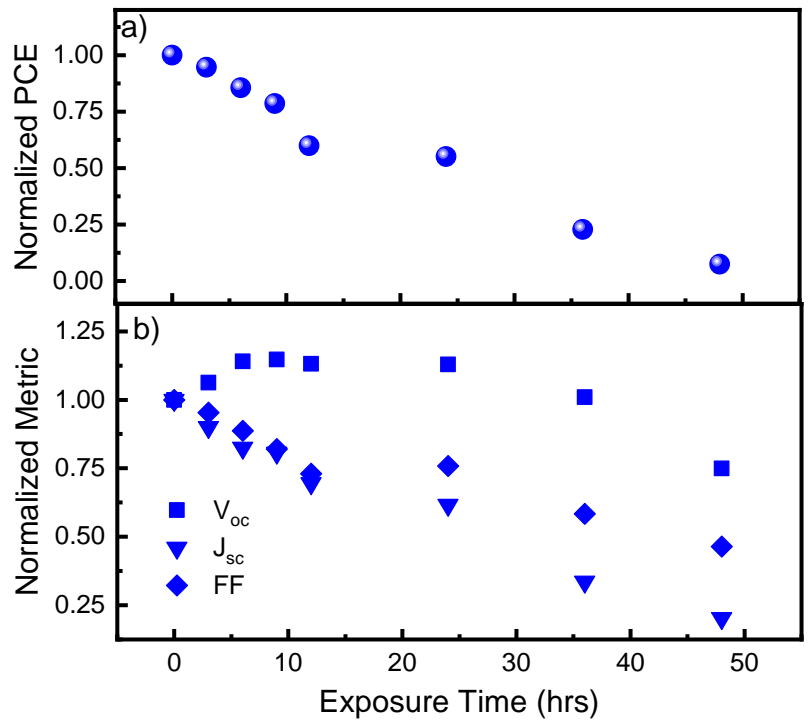

Figure S12 Degradation of nBA-MAPI device performance metrics during exposure to $78 \%$ RH. (a) shows the decrease in the power conversion efficiency, while (b) shows the decrease in the $\mathrm{V}_{\text {oc }}, \mathrm{J}_{\mathrm{sc}}$, and FF over the same time. Results are adapted from ref. 1.

\section{Supporting References:}

1. Wygant, B. R.; Ye, A. Z.; Dolocan, A.; Vu, Q.; Abbot, D. M.; Mullins, C. B. Probing the Degradation Chemistry and Enhanced Stability of 2D Organolead Halide Perovskites. J. Am. Chem. Soc. 2019, 141, 18170-18181. https://doi.org/10.1021/jacs.9b08895. 